\title{
Entry and fireball models vs. observations: What have we learned?
}

\author{
By MORDECAI-MARK MAC LOW ${ }^{1,2} \dagger$ \\ ${ }^{1}$ Astronomy \& Astrophysics Center, University of Chicago, 5640 South Ellis Avenue, Chicago, \\ IL 60637, USA \\ ${ }^{2}$ Also Department of Astronomy, University of Illinois at Urbana-Champaign
}

This review attempts to give a coherent explanation of the main observations of the entry Comet Shoemaker-Levy 9 and the aftermath of the resulting explosions by using models of the tidal breakup of the comet, the entry of individual fragments into the jovian atmosphere, and the resulting fireballs and plumes. A critical review shows that the models appear reasonably well understood. The biggest theoretical uncertainties currently concern how to best tie models of the entry to models of the resulting fireballs. The key unknown before the impact was the size and kinetic energy of the comet fragments. The evidence now available includes the behavior of the chain of fragments, the luminosity of the observed visible fireballs and later infrared emission, the chemistry of the spots, and the lack of seismic waves or perturbations at the water cloud pressure level. These observations point to the fragments having diameters under a kilometer, densities of order $0.5 \mathrm{~g} \mathrm{~cm}^{-3}$, and kinetic energies of order $10^{27} \mathrm{erg}$.

\section{Introduction}

In this review and in the review by Zahnle (this volume; hereafter "the plume review"), we make the argument that the fragments of Comet Shoemaker-Levy 9 that hit Jupiter were quite small, with diameters of under a kilometer and densities of order $0.5 \mathrm{~g} \mathrm{~cm}^{-3}$. The largest fragments probably had kinetic energies of order $10^{27}$ ergs.

The evidence for small impactors includes the length of the pre-impact chain of fragments, the dim entry flashes and fireballs observed at optical wavelengths from Galileo and $H S T$, the lack of observed seismic waves, and the strength and duration of infrared emission from the reentry of the ejected plumes. The evidence for explosions above the water clouds includes the high carbon to oxygen ratio seen by the Hubble Space Telescope (HST) in the ejecta spots (see the review by Lellouch in this volume for discussion of the $\mathrm{CO}$ observations, which, although they yield a lower $\mathrm{C} / \mathrm{O}$ ratio, apparently still do not indicate penetration of the water clouds), and the lack of perturbations in $3 \mathrm{~cm}$ radio observations penetrating through to the 5 bar level where the water clouds are expected to lie. Plume heights do not serve as good evidence, as I will show below that they are difficult to calibrate.

Chronologically, this review covers the period from the tidal breakup of the parent body on its final passage through perijove through a time about 30 minutes after impact when the last bounce in the IR light curve was detected, excluding the main peak in the IR light curve, which is discussed by the plume review.

\section{Tidal breakup}

Several different groups have modeled the initial tidal breakup of the comet. It determines the size and density of the impacting comet fragments, giving initial conditions for models of atmospheric entry. For this reason I consider these models in some detail.

$\dagger$ Present Address: Max-Planck-Institut für Astronomie, Königstuhl 17, D-69117 Heidelberg, Germany 
The maximum stress on the comet as it passed through perijove was minuscule, so Scotti and Melosh (1993) explained both the low strength and the uniform visual magnitude of SL9's debris by suggesting that 21 gravitationally-bound, primordial "cometesimals" came apart at perijove in a "delta function" breakup, and that each object then followed a post-perijove trajectory independent of the rest. Tidal effects are reduced in their model to a single moment when interparticle forces (such as self-gravity and collisions) are switched off. They integrated cometesimal orbits from perijove to the time of the first observations to derive an initial comet diameter $\approx 1.6 \mathrm{~km}$. They neglected self-gravity, so their model does not constrain density except to imply that the comet was deep inside the Roche limit. Their model has difficulty explaining the historical record of crater chains on Ganymede and Callisto, since longer chains contain larger craters (Schenk et al. 1995). This would imply that larger comets formed from larger cometesimals, in contrast to modern theories of primordial accretion (Weidenschilling 1994).

Sekanina et al. (1994) used a somewhat more sophisticated model, outlined in Sekanina's chapter in this volume. In this model, the comet is assumed to break up during perijove passage. The fragments then remain in a collisionally interacting pile or cloud that produces a distribution of particle sizes before drifting apart. However, they begin to calculate train properties only after an effective time of disruption, when the fragments begin to disperse. Their Figure 8 shows that the derived radius of the parent body in their model explicitly depends on the value adopted for the effective time of disruption.

In essence, this is also a "delta function" breakup model, with the time of breakup equal to their effective time of disruption. Similarly to Scotti \& Melosh (1993), Sekanina et al. neglect self-gravity, although they do not assume that the parent comet consisted of 21 uniform cometesimals. They constrain the comet's rotation by comparison with the observed position angle of the fragment chain. If breakup occurs at perijove, they find the same small parent comet diameter as Scotti and Melosh (1993). However, they instead propose that the effective time of breakup was more than 2 hours after perijove, allowing them to find solutions more consistent with the $7 \mathrm{~km}$ diameter parent comet proposed by Weaver et al. (1994).

The assumption of a single moment of breakup or dispersion, however, oversimplifies the process. Sekanina et al. (1994) are correct in assuming that the comet will begin to be affected by the strain well before the final dispersion. However their assumption that the comet remains spherical appears incorrect. The tidal forces distort the body into a cylinder in a process that begins hours before perijove and continues for hours after. The distortion begins with the initial strain, followed by torquing of the distorted body by planetary tides. The resulting spin-up causes further distortion, as do tides acting across the increasingly elongated shape. Finally the fragments of the comet lose physical contact with each other, and finally disperse or clump due to self-gravity, depending on the ratio of self-gravity to tides. Asphaug and Benz (1994) used an N-body code incorporating self-gravity and collisions to demonstrate that the 21-grain "cometesimal" model of Scotti and Melosh (1993) cannot fit the observations. Self-gravity results in pairs or larger clusters of cometesimals sticking together for densities greater than $0.05 \mathrm{~g} \mathrm{~cm}^{-3}$, so the resulting chain contains far fewer than 21 observable fragments.

On the other hand, Asphaug \& Benz (1994) used Dobrovolskis' (1990) analysis of gravity and strength in tidal encounters to show that the comet was effectively strengthless when it encountered Jupiter. For tides to fragment an intact object into $\sim 21$ pieces, the maximum body strength must have been lower than the peak stress at perijove by

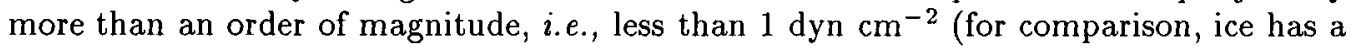



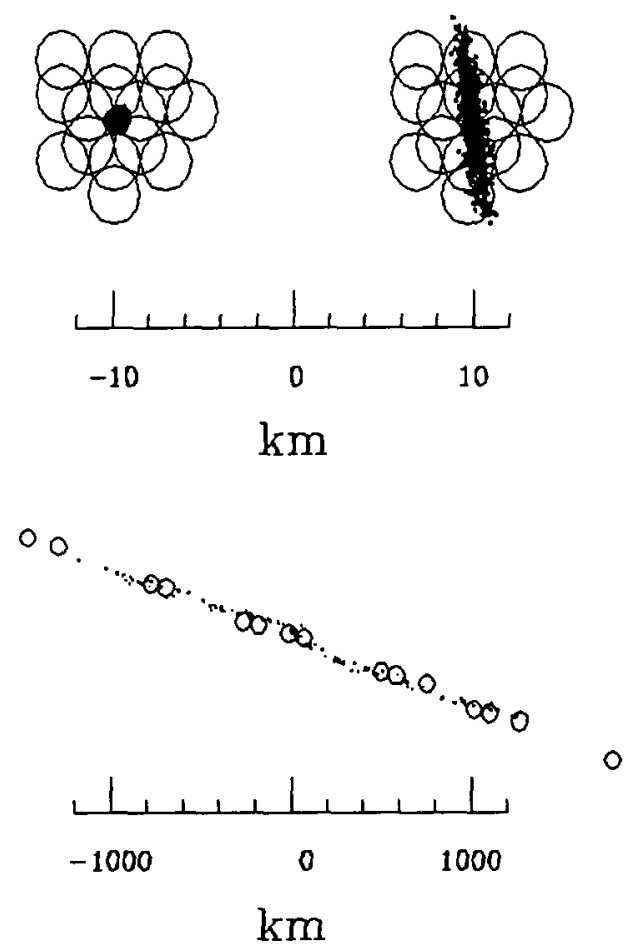

FIGURE 1. Comparison between evolution of a strengthless comet held together by self-gravity (Asphaug \& Benz 1994) and evolution of a 21-grain, $9 \mathrm{~km}$ comet that breaks up instantaneously at a fixed time after perijove (following Sekanina et al. 1994). The first panel shows initial conditions. The second panel shows both models at the time of breakup. Note that, at this time, the length of the strengthless chain is equal to the diameter of the 21-grain comet. The final panel demonstrates that both models reach the correct position angle at late times.

strength of about $10^{7} \mathrm{dyn} \mathrm{cm}^{-2}$ ). This suggests the parent comet consisted of a number $N \gg 21$ of small particles interacting primarily through gravitational forces.

Asphaug \& Benz (1994) then used their N-body code to show that the observations are best fit by a parent comet between 1.5 and $2.0 \mathrm{~km}$ in diameter, consisting of hundreds or thousands of grains. Although particles of fixed size were used for computational convenience, the results do not vary whether hundreds or thousands of particles are used in the computation, and so should not vary substantially if a realistic distribution of particle sizes is used. Figure 1 compares the development of such a comet to the development of a comet that breaks up at a fixed time after perijove. The bulk density of the parent body can be constrained to lie between 0.5 and $0.7 \mathrm{~g} \mathrm{~cm}^{-3}$, because the number of clumps (fragments) observed in the chain is sensitive to density, due to the action of self-gravity.

Asphaug \& Benz's numerical results agree with the analytical tidal breakup model of Sridhar \& Tremaine (1992). These results were independently and almost simultaneously arrived at by Solem (1994), and were recently verified by Richardson, Asphaug, \& Benner (1995) who use the exact orbit of the comet (including aspherical moments of the jovian field plus the influence of Saturn and the Sun) to follow the fragments until they arrive back at Jupiter two years later. The model appears robust. The discrepancy of comet diameter and position angle between the model of Sekanina et al. (1994) and the selfgravitating cluster models (Asphaug \& Benz 1994, Solem 1994, Richardson et al. 1995) 
was resolved by Asphaug \& Benz (1995). They demonstrate that, by the time Sekanina et al. allow breakup to begin (more than 2 hours after perijove), the $1.5 \mathrm{~km}$ diameter comet used by Asphaug \& Benz has deformed into a $9 \mathrm{~km}$ long "cigar" (see Fig. 1) with a rotation period resulting from planetary torque that matches the rotational period that Sekanina et al. found for their $9 \mathrm{~km}$ diameter comet from observational constraints.

An analytic explanation has been advanced for the sensitivity of the number of the clumps to the density (Hahn \& Rettig 1995). After breakup, comet material is distributed more or less uniformly along a cylinder. It then clumps due to Jeans instabilities driven by self-gravity. To get the observed number of fragments, assuming coagulation occurs when the cylinder crosses the Roche distance of the original parent, a bulk density of $0.6 \mathrm{~g} \mathrm{~cm}^{-3}$ is required, in good agreement with the numerical models.

A final point in favor of accepting the smaller comet is that it happens to agree with the median diameter derived from the record of crater chains on Ganymede and Callisto by Schenk et al. (1995).

\section{Early stages of entry}

Consider the first two peaks in the generic, ground-based, IR light curve, described by Nicholson (this volume) as P1 and P2. P1 appears to be caused by the meteor trail resulting from the initial entry of a comet nucleus, while P2 results from the appearance of the resulting fireball over the horizon as viewed from the Earth (see Fig. 2 of the plume review). A few nuclei showed a very faint precursor to the precursors that we could label P0, slowly rising for as much as a minute before P1. The form of P0, and its lack of detection by Galileo, suggest that it is produced by the massive meteor shower that occurs as the tidally stretched coma enters before the central nucleus.

The luminosity of the meteor trail can be estimated by considering the flight of the impactor through the upper jovian atmosphere. Graham et al. (1995) showed this to be a plausible explanation for P1 by assuming that drag dominated energy transfer to the atmosphere. At these very high altitudes, however, radiative ablation actually dominates the energy transfer. The mass loss due to radiative ablation of a spherical comet with radius $r_{c}$ entering with velocity $v_{c}$ is

$$
\frac{d m_{c}}{d t}=-\frac{C_{H}}{2 Q} \pi r_{c}^{2} \rho(z) v_{c}^{3}
$$

where $C_{H}$ is the heat transfer coefficient, $\rho(z)$ is the atmospheric density, and $Q$ is the heat of ablation. The energy loss rate is then

$$
\begin{aligned}
\frac{d E}{d t} & =\frac{1}{2} \dot{m}_{c} v_{c}^{2}=-\frac{C_{H}}{4 Q} \pi r_{c}^{2} \rho(z) v_{c}^{5} \\
& =-\left(10^{31} \mathrm{erg} \mathrm{s}^{-1}\right) \rho(z)\left(\frac{r_{c}}{200 \mathrm{~m}}\right)^{2}\left(\frac{C_{H}}{0.1}\right)\left(\frac{v_{c}}{60 \mathrm{~km} \mathrm{~s}^{-1}}\right)^{5}
\end{aligned}
$$

where $Q=2.5 \times 10^{10} \mathrm{erg} \mathrm{g}^{-1}$ for an icy comet (Chyba et al. 1993). This is a factor of forty higher rate than Graham et al. (1995) used. However, the efficiency of conversion of this energy to visible radiation $\eta$ remains unknown. Small meteors in the Earth's atmosphere have efficiencies $\eta \sim 10^{-4}-10^{-2}$ (Bronshten 1983); larger objects may be somewhat more efficient as longer pathlengths increase the optical depth through hot gas. Graham et al. found that the peak flux of the first flash for the R impact was $\sim 0.4 \mathrm{Jy}$ at $2.3 \mu \mathrm{m}$, corresponding to $5 \times 10^{19} \mathrm{erg} \mathrm{s}^{-1}$. The detected trail would then require an atmospheric density $\rho=\left(5 \times 10^{-12} \mathrm{~g} \mathrm{~cm}^{-3}\right) \eta$. For an efficiency of $\eta=0.01$ this corresponds to an altitude of $300 \mathrm{~km}$ above the 1 bar level, $1.4 \mathrm{~s}$ of flight time above the visible limb at 
$240 \mathrm{~km}$. The trail could have been observed longer if the efficiency were higher, or if scattering from dust allowed its observation below the limb. Although the uncertainties are large, it appears plausible that $\mathrm{P} 1$ was indeed produced by the meteor trail through the upper atmosphere.

The most detailed published treatment of the meteor's passage through the deeper atmosphere is by Chevalier \& Sarazin (1994), who attempt to describe the $60 \mathrm{~km} \mathrm{~s}^{-1}$ bow shock in some detail. They consider only the emission from shocked jovian air, neglecting any additional opacity added to the wake of the comet by ablated cometary material. At a pressure level of $100 \mu$ bar and an altitude of $200 \mathrm{~km}$ above the 1 bar pressure level, Chevalier \& Sarazin (1994) find that hydrogen that has passed through a $60 \mathrm{~km} \mathrm{~s}^{-1}$ shock emits lines in the optical and UV. The opacity of atomic hydrogen at these temperatures is quite low, so they find that the lines remain optically thin until pressures increase to about 10 mbar, at an altitude of $100 \mathrm{~km}$. At this level, the densest regions of hot air behind the bow shock become optically thick, and strong continuum radiation appears, with an optical luminosity $L_{\text {opt }} \sim\left(3 \times 10^{22} \mathrm{erg} \mathrm{s}^{-1} \mathrm{~km}^{-2}\right) A_{c}$ depending on the area of the comet nucleus $A_{c}$. Chevalier \& Sarazin (1994) also find that shocked air in the wake will continue to emit in the optical for a few tens of $\mathrm{km}$ above the plunging comet, at a rate of $\sim 5 \times 10^{22} \mathrm{erg} \mathrm{cm}^{-2} \mathrm{~km}^{-1}$.

The optical luminosity from the bow shock increases rather slowly with depth because more and more of the emission comes out in the UV as the shock temperature increases at greater densities (see Table 2 of Chevalier \& Sarazin 1994). The UV pulse detected by the Galileo UVS probably occurred during this period. The optical luminosity reaches $L_{\text {opt }} \sim\left(9 \times 10^{22} \mathrm{erg} \mathrm{s}^{-1} \mathrm{~km}^{-2}\right) A_{c}$ at a depth of $100 \mathrm{~km}$ below 1 bar. The effective bolide diameter increases by a factor of five within a scale height when Rayleigh-Taylor instabilities become effective, as discussed in the next section (Mac Low \& Zahnle 1994). The increase of bow shock area by a factor of 25 in a second probably produces the initial sharp rise in the Galileo $P P R$ optical light curve. The initial peak has $L_{\text {opt }} \sim$ $5 \times 10^{23} \mathrm{erg} \mathrm{s}^{-1}$ (Chapman, this volume). By assuming this factor of five increase in radius at the point of maximum energy release, I can estimate the initial bolide diameter based on the observed $L_{\text {opt }}$. Table 2 of Chevalier \& Sarazin (1994) suggests that the luminosity at a pressure level of 2 bars is $L_{\text {opt }} \sim\left(8 \times 10^{22} \mathrm{erg} \mathrm{s}^{-1} \mathrm{~km}^{-2}\right) A_{c}$. Taking the increase of area into account, the observed value of $L_{\text {opt }}$ suggests the initial diameter of the bolide was just over a half kilometer, supporting the argument for small impactors.

During the meteor phase, the bow shock surrounding the nucleus entering at a velocity $v_{c}$ confines it and compresses it from its original density $\rho_{c}$ to its compressible limit $\rho_{c}^{\prime}$, as shown in Figure 2. Field \& Ferrara (1995) show that this occurs before the onset of the instabilities that ultimately tear the nucleus apart. They do this by directly computing the passage through the nucleus of the compression wave driven by the ram pressure of the bow shock. The front face of the nucleus decelerates to a velocity $v_{c}^{\prime}$, while the rest of the comet continues unaffected until the compression wave reaches it. The thickness of the compressed region $h$ increases as more and more of the nucleus runs into it. The thickness can be found by equating the mass flux out of the uncompressed nucleus to the mass flux into the compressed layer to get

$$
\frac{d h}{d t}=\frac{\rho_{c}}{\rho_{c}^{\prime}}\left(v_{c}-v_{c}^{\prime}\right)
$$

The velocity of the compressed layer can be found from the drag equation (eqn. 4.8) acting on it, so that the compressed layer grows as

$$
h=\frac{H}{\rho_{c}^{\prime}}\left[C_{D} \rho_{c} \rho(z)\right]^{1 / 2} \sec \theta .
$$




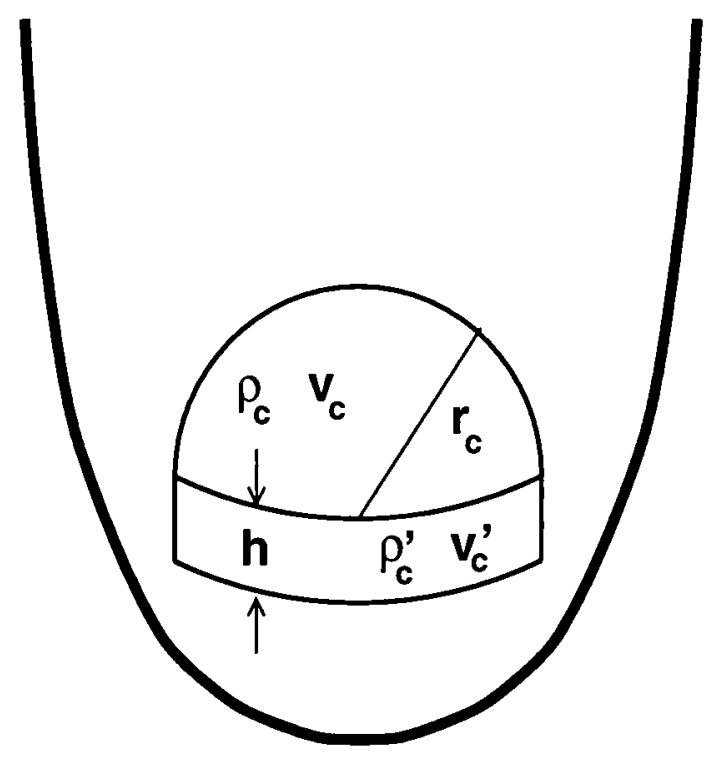

Figure 2. Initial compression of entering comet nucleus by bowshock. This will occur before other processes can affect the nucleus.

The final thickness of the layer, $h_{f}=\left(4 \rho_{c} / 3 \rho_{c}^{\prime}\right) r_{c}$ is reached at an altitude $z_{f}$, where the atmospheric density

$$
\rho\left(z_{f}\right)=\frac{\rho_{c}^{\prime 2} h_{f}^{2}}{C_{D} \rho_{c} H^{2} \sec ^{2} \theta} .
$$

For example, for $\rho_{c}=0.5 \mathrm{~g} \mathrm{~cm}^{-3}, R=300 \mathrm{~m}, \rho_{c}^{\prime}=1 \mathrm{~g} \mathrm{~cm}^{-3}$, and $\theta=45^{\circ}$, the altitude of complete compression is $z_{f}=+50 \mathrm{~km}$, more than a scale height above the level of final energy deposition, as the next section shows.

\section{Final Deceleration and energy deposition}

Where does the energy and mass of the incoming bolide get deposited in the atmosphere? The subsequent development of the fireball, partition of energy between the lower atmosphere and plume, and distribution of ejecta all depend on the initial conditions set up during this final entry phase. A number of different groups have used analytic and numerical models to try to answer these questions.

This section begins by describing the three main analytic approaches-ablation models, pancake models, and an instability model. I critique the simple ablation models, and then show that the pancake models succeed as well as they do because they are equivalent, to within factors of order unity, to the more physically detailed instability model. I then discuss the numerical models, why they disagree with each other, and attempt to show which ones are most reliable. Taking proper account of the continuing downward movement of the wake results in an altitude of peak energy deposition about half a scale height below the point of peak instantaneous energy deposition, the quantity predicted by the analytic models.

\subsection{Analytic models}

The first model published (Sekanina 1993) computed the energy deposition of a nucleus by following its radiative ablation. The mass loss due to radiative ablation is given by equation (3.1). Sekanina (1993) took his ablation coefficient (equivalent to $C_{H} / Q$ 


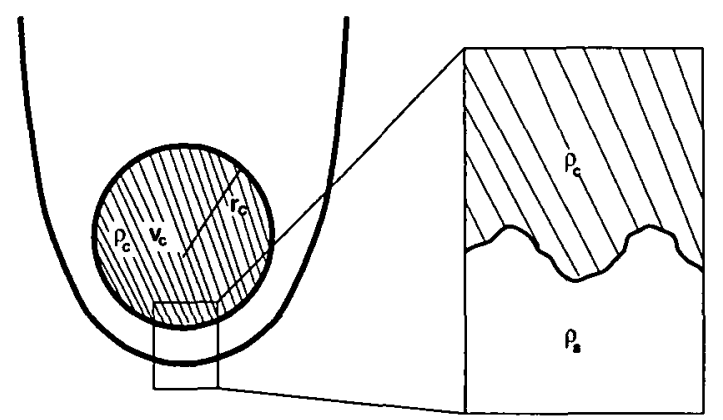

Figure 3. Growth of Rayleigh-Taylor instabilities along the front surface of an entering comet nucleus.

in eqn. [3.1]) from observations of terrestrial fireballs, getting an effective heat transfer coefficient $C_{H}=0.5$, rather larger than the value usually assumed for meteors of $C_{H}=$ $0.1-0.2$ (Bronshten 1983). A constant heat transfer coefficient appears to explain observations of terrestrial meteors with diameters up to meters. Sekanina (1993) assumed that kilometer-sized objects would behave in the same way. For the smaller objects, the amount of ablation depends linearly on the flux of kinetic energy, $\rho v^{3} / 2$, across the shock. However, radiative ablation of larger objects is limited by thermal emission from the hot, postshock gas, proportional to $\sigma T_{s}^{4}$ (Biberman et al. 1980, Zahnle 1992). This does not increase as fast as $\rho$ as the object moves deeper. Chevalier \& Sarazin (1994) reach similar conclusions. Field \& Ferrara (1995) go further by attempting to analytically model the advection of the vaporized surface layer. They conclude that even the rate given by Zahnle (1992) is an overestimate for these large objects because of the need to not only vaporize material off the surface, but also to decelerate it. For example, a nucleus with radius $r_{c}=1.5 \mathrm{~km}$, and mass $m_{c}$ loses only $3 \times 10^{-3} m_{c}$ as it falls through a scale height at the 1 bar pressure level.

The entering nucleus instead gets torn apart by the ram pressure from the bowshock. For large objects, this process determines the energy deposition profile. Energy gets transferred more efficiently to the atmosphere as the cross-section of the nucleus increases due to fragmentation. Fragmentation occurs because low-density, shocked gas decelerates the high density nucleus, causing the front of the nucleus to become Rayleigh-Taylor unstable as shown in Figure 3. (Note that, though Kelvin-Helmholtz instabilities occur, as argued in the chapter by Crawford, they grow about four times slower than the Rayleigh-Taylor instabilities, and so do not dominate the spreading of the impactor.) Svetsov, Nemtchinov \& Teterev (1995) have analytically modeled the fragmentation due to Rayleigh-Taylor instabilities as follows.

The growth rate for these instabilities is (e.g., Chandrasekhar 1961, p. 435)

$$
\omega=\left(\frac{2 \pi a}{\lambda}\right)^{1 / 2}\left(\frac{\rho_{c}-\rho_{s}}{\rho_{c}+\rho_{s}}\right),
$$

where $a$ is the acceleration of the interface, $\lambda$ is the unstable wavelength under consideration, $\rho_{c}$ is the density of the comet nucleus, and $\rho_{s}$ is the density of the shocked gas. As long as $\rho_{c} \gg \rho_{s}$ the second factor on the right (the Atwood number) approaches unity. The acceleration can be derived from the drag equation,

$$
a=\frac{C_{D} \pi r_{c}^{2} \rho(z) v^{2}}{2 m_{c}}
$$

where the drag coefficient $C_{D}$ is measured to be close to unity for a sphere, the atmospheric density at altitude $z$ is $\rho(z)$, and the velocity is $v_{c}$. The relevant dynami- 


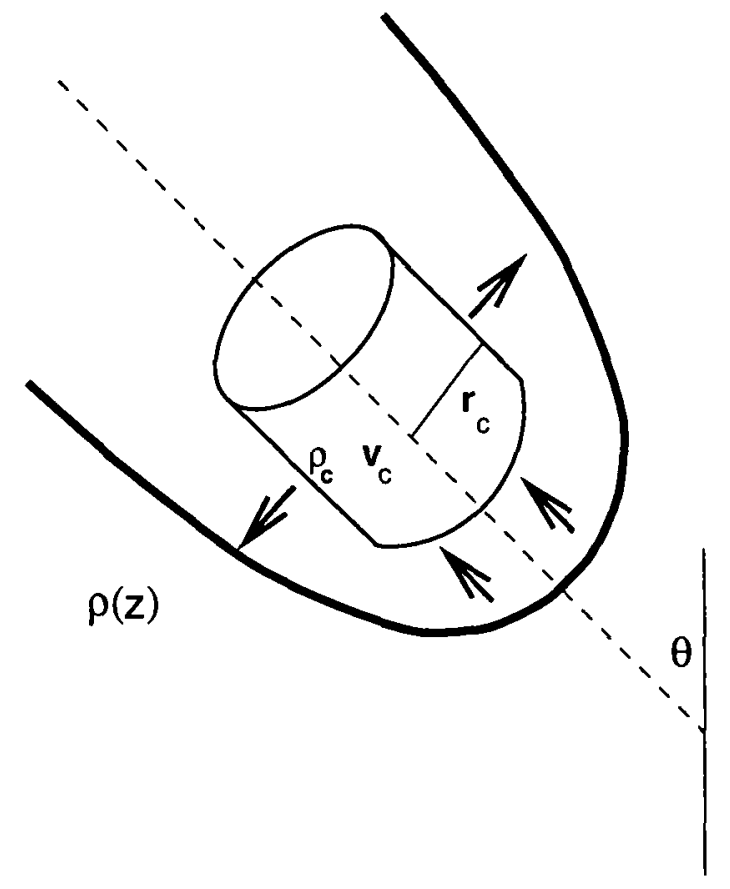

Figure 4. Pancake model: Quasistatic deformation of entering nucleus by the difference in ram pressure acting on the front and the side of the object.

cal timescale $t_{\text {dyn }} \sim H \sec \theta / v_{c}$. While the smallest wavelengths grow the fastest, the wavelengths that fragment the bolide have wavelength $\lambda \sim r_{c}$. When the growth rate corresponding to that wavelength $\omega(\lambda, t)$ increases to the point that $\omega t_{\mathrm{dyn}}=1$ the comet fragments, causing the energy deposition rate to increase dramatically. In fact, this is a simplification of the true physics, since shorter wavelengths that grow into the nonlinear regime begin to combine into longer wavelength perturbations (Read 1984, Youngs 1984), explaining why higher resolution is needed in gas dynamical computations to properly model the fragmentation. The atmospheric density at which fragmentation occurs is given by

$$
\rho(z)=\frac{4}{3 \pi C_{D}}\left(\frac{r_{c}}{H} \cos \theta\right)^{2} \rho_{c},
$$

where $H$ is the scale height of the atmosphere.

A number of workers have modeled the entry and energy deposition profile using an even simpler model first proposed by Zahnle (1992) that has come to be called the pancake model (Zahnle \& Mac Low 1994, Mac Low \& Zahnle 1994, Chevalier \& Sarazin 1994, Field \& Ferrara 1995, Svetsov et al. 1995). The assumption used in this model is that once aerodynamic forces have overcome any material strength, the incoming object quasistatically deforms due to the difference in the ram pressure acting on the front and the sides, as shown in Figure 4. As the object flattens, its cross-section and its drag increase, bringing it to a rather abrupt halt accompanied by explosive energy release. The most elegant formulation of this model is given by Field \& Ferrara (1995), who took a classical mechanical approach by deriving an equation of motion from Lagrange's equation. They draw the analogy to the response of a fluid in a dish when the sides of the dish are removed. Zahnle \& Mac Low (1994) followed Chyba, Thomas \& Zahnle (1993) in adding a term to account for radiative ablation in the upper atmosphere, and solving 
the resulting equation numerically. Here I present the simplest version of the pancake model in order to give analytic results.

The entering object is taken to be a right circular cylinder of radius $r_{c}$, density $\rho_{c}$, and height $h$, entering with initial velocity $v_{c}$, at an angle $\theta$, as shown in Figure 4 . The drag coefficient of a right circular cylinder is measured to be $C_{D}=1.7$. The ram pressure on the front face $P_{f}=\frac{1}{2} C_{D} \rho(z) v^{2} \sec ^{2} \theta$, where $v$ is the instantaneous velocity. The average internal pressure $P_{i} \sim \frac{1}{2} P_{f}$. It acts on the sides of the cylinder causing it to expand, so the force equation is

$$
2 \pi r_{c} h P_{i}=\pi r_{c}^{2} h \rho_{c} \ddot{r}_{c}
$$

where the right-hand-side is the mass of the cylinder times its sideways acceleration. Converting from time derivatives to space derivatives, $d / d t=v \sec \theta(d / d z)$, and expanding, we find that the radius evolves as

$$
r_{c} \frac{d^{2} r_{c}}{d z^{2}}+\frac{r_{c}}{v} \frac{d v}{d z} \frac{d r_{c}}{d z}=\frac{C_{D} \rho(z)}{2 \rho_{c}} \sec ^{2} \theta
$$

The nonlinear term may be neglected to first order. In an exponential atmosphere, this equation may then be approximately solved,

$$
r_{c}(z) \simeq H \sec \theta\left(\frac{2 C_{D} \rho(z)}{\rho_{c}}\right)^{1 / 2}
$$

This is effectively equation (4.9), derived by Svetsov et al. (1995) by considering RayleighTaylor instabilities. This agreement accounts for the surprisingly good performance of the pancake model when compared to numerical models (Mac Low \& Zahnle 1994).

Substituting equation (4.12) into the drag equation (4.8) and integrating, the velocity of the impactor

$$
v(z)=v_{c} \exp \left(-\frac{\pi C_{D}^{2} \rho(z)^{2} H^{3} \sec ^{3} \theta}{2 m_{c} \rho_{c}}\right) .
$$

The energy release rate neglecting ablation is $d E / d z=m v d v / d z$, so the pressure level of maximum energy release is

$$
P_{0}=(9.0 \text { bars }) C_{D} d_{\mathrm{km}}^{3 / 2}\left(\frac{\rho_{c}}{1 \mathrm{~g} \mathrm{~cm}^{-3}}\right)\left(\frac{g}{2486 \mathrm{~cm} \mathrm{~s}^{-2}}\right)\left(\frac{H}{45 \mathrm{~km}}\right)^{-1 / 2}\left(\frac{\mathrm{sec} \theta}{\sqrt{2}}\right)^{-3 / 2}
$$

where $g$ is the acceleration of gravity, and $d_{\mathrm{km}}$ is the diameter of the comet, measured in $\mathrm{km}$. The jovian atmosphere actually does not have a constant scale height, so this equation should be solved iteratively, starting with a guess at the scale height in the region of maximum energy deposition, and then using on subsequent iterations the scale height $H$ corresponding to the pressure level $P_{0}$ given by the previous iteration. Below, I will show that numerical models suggest that the final level of energy deposition is about half a scale height deeper than the instantaneous level given here, which should also be taken into account when using this equation. Figure 5 shows the height of maximum instantaneous energy deposition for impactors of different diameter and density. including the effects of high-altitude radiative ablation as described in Zahnle \& Mac Low (1994), which shifts the curves slightly from the purely analytic solution.

\subsection{Numerical models}

In contrast to the analytic models, numerical models of the entry of a comet nucleus have come to a remarkably broad range of conclusions. A nucleus with diameter of $1 \mathrm{~km}$, and density $1 \mathrm{~g} \mathrm{~cm}^{-3}$, has been predicted to explode everywhere from the stratosphere to well below the water clouds. I explain the differences by examining the numerical 


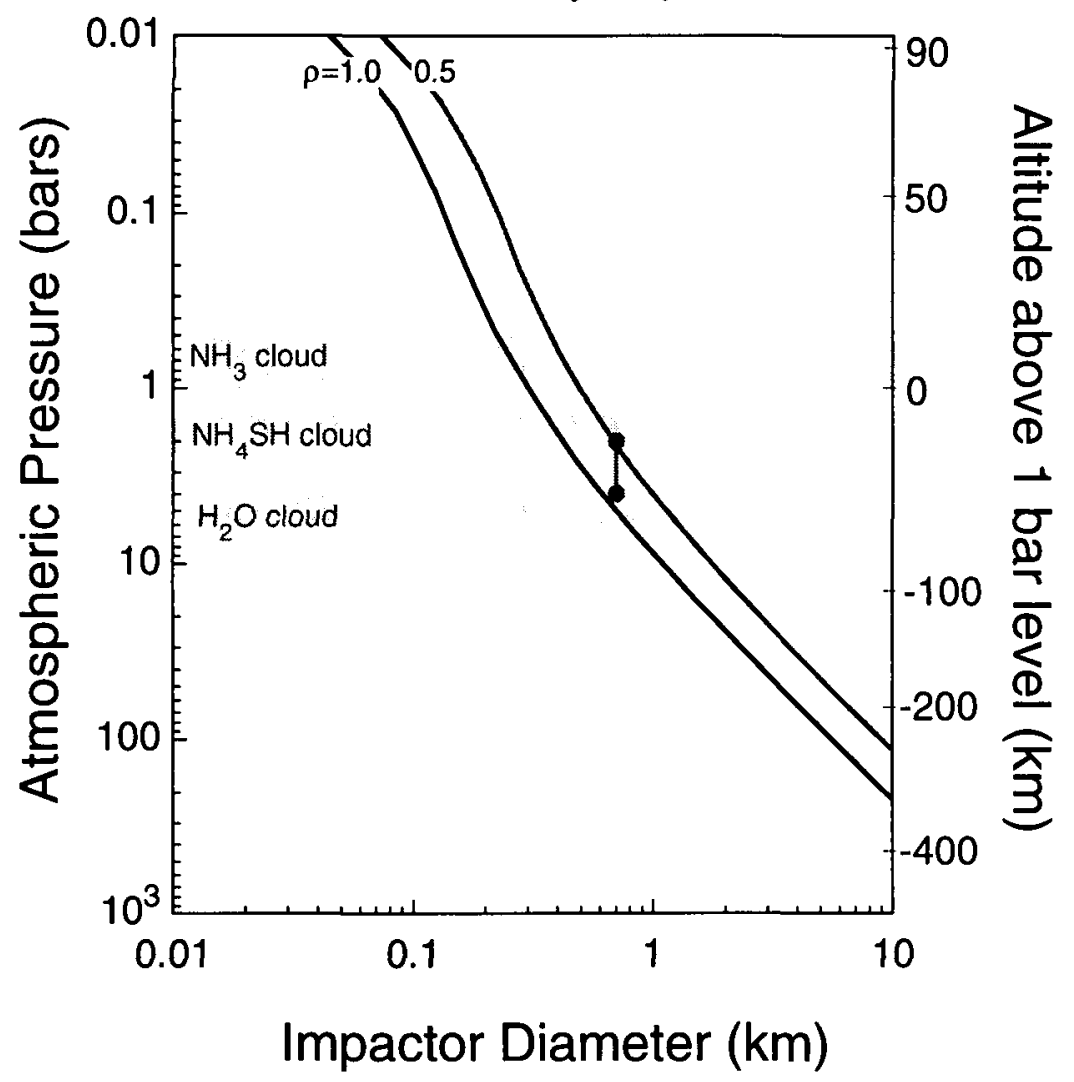

FIgURE 5. Altitude of maximum instantaneous energy deposition for impactors of density 0.5 and $1.0 \mathrm{~g} \mathrm{~cm}^{-3}$ as a function of impactor diameter, using the model described in Zahnle \& Mac Low (1994). The top of the dumbbell-shaped point shows the height of instantaneous energy deposition of an impactor of the size described by Asphaug \& Benz (1994), while the bottom shows the height of final energy deposition after downward advection (see text).

methods, grid resolution, and diagnostic measurements used. It appears that downward advection of the wake lowers the height of maximum energy deposition about half a scale height below the instantaneous value given by the analytic models.

\subsubsection{Critical review}

I divide the models into four categories. First, are those groups that show maximum energy deposition at or above the predictions of the pancake model, including Mac Low \& Zahnle (1994), Yabe et al. (1994), and Shoemaker, Hassig, \& Roddy (1995). I also include in this category the computations of shocked clouds of interstellar gas by Klein, McKee \& Colella (1994) that have been independently analyzed using a model equivalent to the pancake model. Second, the Sandia group (Boslough, Crawford, Trucano, and Robinson) has converged on a prediction of energy deposition about a scale height deeper than the analytic model in a series of important papers (Boslough et al. 1994, 1995; Crawford et al. 1994, 1995). Third, two groups predicted deep penetration, with a smooth energy deposition profile having no clear point of maximum energy release (Takata et al. 1994; Wingate, Hoffman, \& Stellingwerf 1995). Finally, two groups presented preliminary results but did not pursue the problem far enough to come to solid conclusions, so I will not discuss them further (Vickery 1993; Moran \& Tipton 1993). 
First, I discuss the models that give results at or above the prediction of equation (4.14). I will discuss my own models more extensively below, but briefly describe here three other models. Hassig, Roddy \& Shoemaker (1995) used a multi-material code that attempts to follow the fragmentation and vaporization of a solid body. Although, due to computational expense, they only followed the last $100 \mathrm{~km}$ or so of the entry path, starting at $31 \mathrm{~km}$ below 1 bar, they appear, with this rather different method, to get results in agreement with equation (4.14). Yabe et al. used a third-order, Eulerian code with a maximum resolution of 30 zones across the radius (I will hereafter denote the resolution by the notation $\mathrm{R} n$, where $n$ is the number of resolution elements in the radius of the object, so the effective resolution here, for example, is R30). They tested several equations of state, and, in agreement with the Sandia group and Mac Low \& Zahnle (1994) found little dependence. Their quantitative result-explosion at 10 bar for a $3 \mathrm{~km}$ diameter comet-is significantly higher than the 75 bar prediction of the pancake model (eq. 4.14) for their parameters. They did not use a realistic jovian atmosphere, instead using one with a constant scale height of $43 \mathrm{~km}$ and a comet hitting at normal incidence rather than an angle of $45^{\circ}$; however it is not clear why that should cause their simulations to show such high explosions. This result needs further examination.

An interesting point of comparison is to models of clouds of interstellar gas hit by supernova blast waves. This is a question of some interest for the state of the interstellar medium and has been attacked vigorously with analytic and computational methods. Klein et al. (1994) used an adaptive mesh refinement technique on an Eulerian grid to achieve effective resolutions as high as R240 on this problem. They performed a careful resolution study to ensure that they only quoted converged results. They independently derived an analytic model of the acceleration of the cloud by the blast wave that is equivalent to Zahnle (1992), as shown in Mac Low \& Zahnle (1994), and showed that it fit their numerical results well.

Next let me discuss the computations performed by the Sandia group (Boslough et al. 1994, 1995; Crawford et al. 1994, 1995). They computed their entry models on an Eulerian grid large enough to capture the entire entry wake. To do this, they placed a moving region of fine zones at the position of the nucleus, and used a ratioed grid with slowly increasing zone sizes along the tail. By this method, they achieved resolutions of R25 to R50 at the nucleus, sufficient to capture its breakup, according to the resolution study done by Mac Low \& Zahnle (1994). The resolution slowly degraded along the wake, with zone sizes reaching $5 \mathrm{~km}$ at a distance of $100 \mathrm{~km}$ (Crawford et al. 1995) and $25 \mathrm{~km}$ at the lowest resolution. The advantage of this grid is that they were able to directly measure energy deposition in the atmosphere at the end of their computation, taking account of the downward advection of energy in the moving wake swept up by the nucleus. In contrast, the pancake model computes the instantaneous loss of kinetic energy from the cometary material; that was also the quantity measured by Mac Low \& Zahnle (1994) and Yabe et al. (1995). I'll show below that this explains part of the difference between their reported results and those of Mac Low \& Zahnle (1994).

There was some confusion about how deep the Sandia group actually predicted maximum energy deposition for a $1 \mathrm{~km}$ object. This appears to have occurred because, in their first papers (Crawford et al. 1994, Boslough et al. 1994) they reported results for an object with a 100 bar yield strength (appropriate for a solid, stony asteroid) that deposited its energy at $180 \mathrm{~km}$. However, later they quoted results for a strengthless, but incompressible, object probably more appropriate for the rubble piles suggested by Asphaug \& Benz (1994), that deposited its energy at 120-130 km (Crawford et al. 1995).

Third, I discuss the models that found deep penetration and smooth energy deposition profiles. Takata et al. (1994) used a smooth particle hydrodynamics (SPH) code. SPH 
follows the fluid with particles, and computes intensive quantities such as density and pressure by averaging over the particles. Although it appears able to reach arbitrary resolution, in practice a minimum particle separation or smoothing length must be enforced to avoid extremely small timesteps and excessive computation time. Takata et al. used a smoothing length of $0.25 R_{c}$, giving an effective resolution of only $\mathrm{R} 4$. This resolution cannot resolve the Rayleigh-Taylor instabilities that bring the comet to an abrupt halt as discussed above. MacLow \& Zahnle (1994) showed computations on an Eulerian grid at a resolution of $\mathrm{R} 6$ that behaved very similarly to the models shown in Takata et al. and showed that the behavior changes at higher resolution, where it converges on a different solution.

Wingate et al. (1995) also used an SPH code, but at much higher resolution, with effective resolutions reaching $\mathrm{R} 50$, easily sufficient to resolve the instabilities. Their computation apparently suffered from a more subtle problem, however. Because of the stochastic nature of SPH particles, defining the exact position of a shock front is difficult. As a result, shocks tend to be much broader than in grid-based codes of similar resolution. A shock front is, of course, stable against Rayleigh-Taylor instabilities, although the contact discontinuity between the shocked gas and the comet nucleus is unstable. In the computations shown by Wingate et al. (1995), the contact discontinuity was (at least intermittently) stable, probably because the shock was so broad that it sometimes overlapped the contact discontinuity and stabilized it. As a result, the nucleus penetrated much deeper than it would have otherwise. This may also explain the strong oscillations they observed, if the shock moved on and off the contact discontinuity.

\subsubsection{Computations}

For my own computations described here, I used ZEUS, a general purpose astrophysical MHD code, developed by M. L. Norman and his students at the Laboratory for Computational Astrophysics (LCA) of the National Center for Supercomputing Applications. A full suite of test problems is described by Stone \& Norman (1992). The particular version of the code used here is called ZEUS-3D, and was developed by Norman and D. A. Clarke. It is publically available by registration with the LCA at Ica@ncsa.uiuc.edu. The code uses second-order Van Leer (1977) advection on an Eulerian, moving grid in Cartesian, cylindrical or spherical geometry. The code is fully three-dimensional and includes magnetic fields, but for reasons of time we have only done two-dimensional, gas dynamical models to date. Shocks are resolved using a Von Neumann artificial viscosity. I have implemented both tracer fields and tracer particles for this problem.

For our models we use two different equations of state. One is just an adiabatic equation of state with adiabatic index $\gamma=1.2$ at early times when dissociation and ionization is important (Chevalier \& Sarazin 1994) or $\gamma=1.4$ for computations extending to later time (e.g., those discussed in the plume review). The other is a stiffened gas equation of state appropriate to ice, described by Mac Low \& Zahnle (1994), which we use for the comet nucleus in our initial entry models. The entry models published in MacLow \& Zahnle (1994) used a cylindrical grid $5 \mathrm{~km}$ high by $3 \mathrm{~km}$ in radius. The innermost $3 \mathrm{~km}$ by $1 \mathrm{~km}$ has the full resolution specified (up to a maximum of R100, corresponding to a zone size of $5 \mathrm{~m}$ ); outside of that there is a layer of zones each a factor of 1.03 bigger than the one inside until zones ten times as large as the central zones are reached, and finally a layer of constant-size zones out to the edges of the grid, for a total of 0.2 megazones in our R100 computation.

The energy deposition of these models was determined by measuring the loss of kinetic energy by cometary material on the grid. Figure 6 shows that, measured this way, the numerical models agree rather well with the simple analytic model given by equation (4.14). 


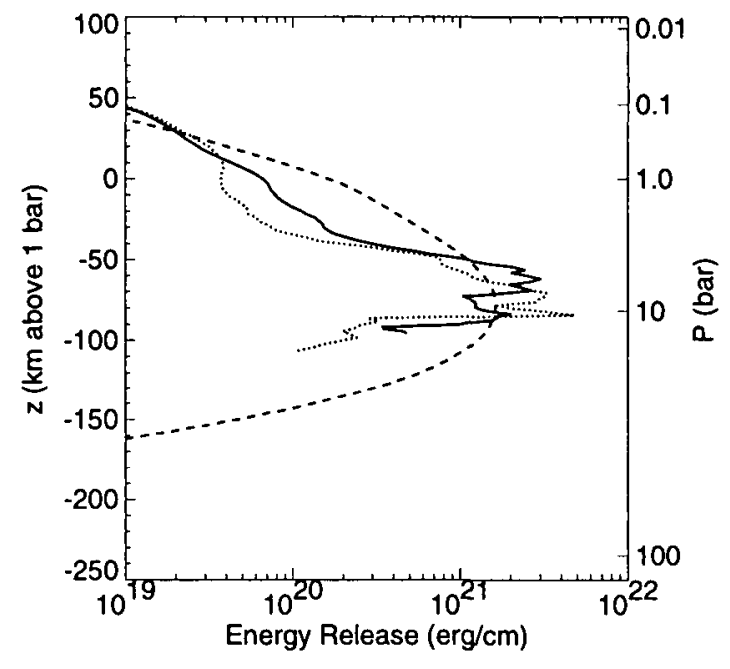

FIGURE 6. Comparison of energy release profiles for incompressible (solid curve) and compressible (dotted curve) equations of state run at a resolution of R50. The simple pancake model of equation (4.14) is also shown (dashed curve), with a drag coefficient $C_{D}=1$, a pressure scale height $H=45 \mathrm{~km}$, and a $1 \mathrm{~km}$ diameter nucleus. From Mac Low \& Zahnle (1994).

However, this method of measurement has two problems. First, cometary material flows off the back of the grid while it still carries some kinetic energy, and second, kinetic energy transferred to the ambient atmosphere is not immediately thermalized, but instead is partitioned between kinetic and thermal energy in the atmosphere. The second effect appears to be far more important, as it causes significant downward advection of energy contained in the ambient atmosphere, as pointed out by the Sandia group.

To measure these two effects, I ran a new R50 computation, but instead of cutting off the wake after $5 \mathrm{~km}$, I extended the region with coarse vertical grids (100 m zones) up for a full $100 \mathrm{~km}$, and the region with coarse radial grids out to $10 \mathrm{~km}$, for a total of 3.1 megazones. For comparison, at $100 \mathrm{~km}$ above the entering nucleus, the Sandia group has vertical zones 50 times as large. I forced the grid to follow the entering comet nucleus until it had fragmented to such an extent that no zone contained more than $90 \%$ comet material, and then allowed material to flow off the bottom of the grid. (In retrospect, this was not the best way to do this-following the downward flow for longer would have been better-but the computation took 40 Cray Y-MP hours so I have not yet run a better model.)

Figure 7 shows the entry wake just after the comet nucleus has passed through the altitude of maximum instantaneous energy deposition. The shape of the wake shows the explosive nature of the energy deposition when the nucleus begins to fragment. The piece of the nucleus seen penetrating deeper into the atmosphere carries about a quarter of the kinetic energy of the initial nucleus. This is an upper limit to the real value, as the deeply penetrating piece is badly underresolved. Comparison between the R50 and R100 models of Mac Low \& Zahnle (1994) suggests that the central fragment gets torn apart more thoroughly than an R50 model shows, and so stops more quickly.

I tested for downward advection of energy as suggested by the Sandia group by integrating the actual thermal energy contained on the grid at several times after the nucleus reached the altitude of maximum energy deposition. The resulting profiles are compared to the instantaneous energy loss from the cometary material in Figure 8. The interpre- 


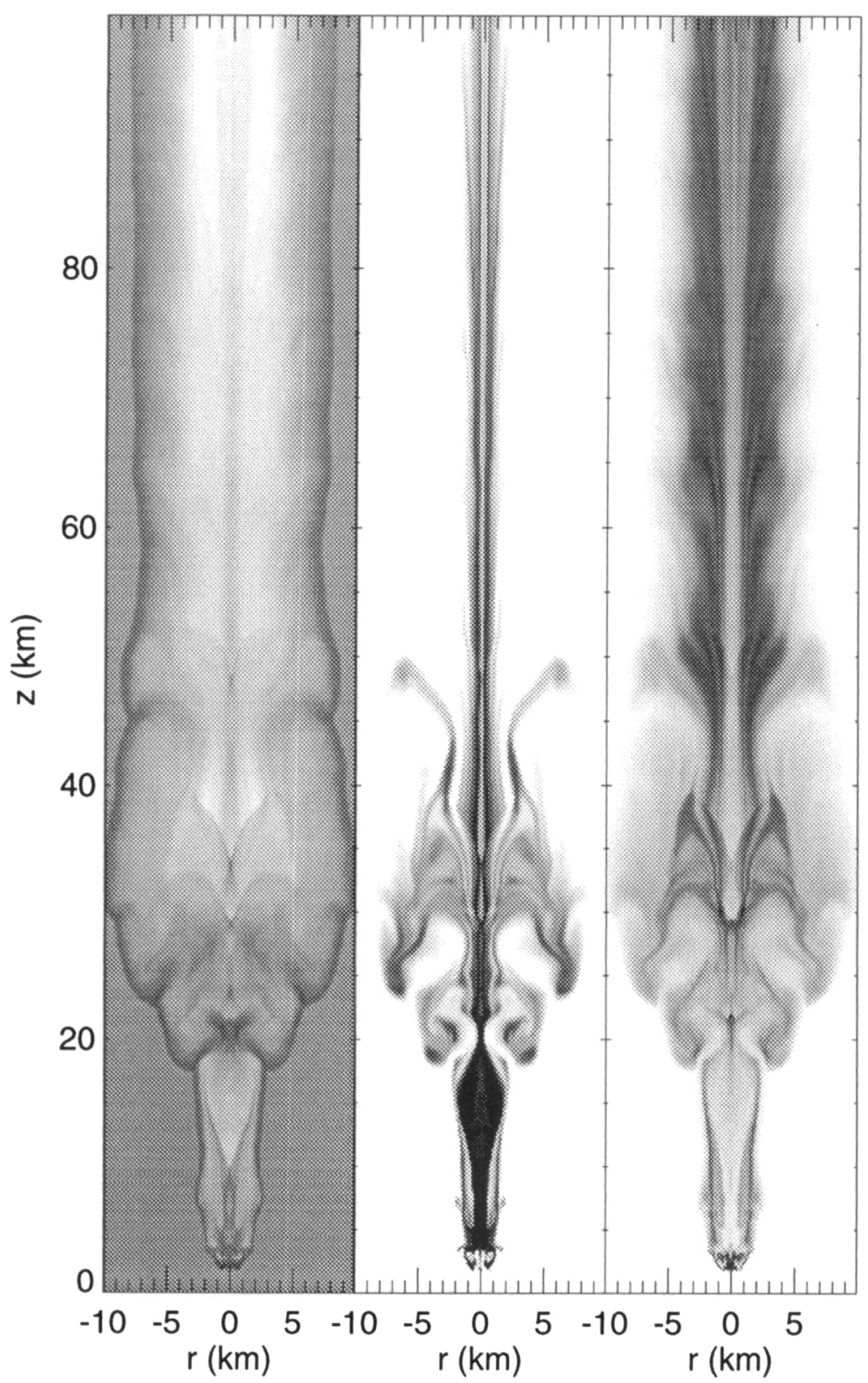

Figure 7. Greyscale images of $100 \mathrm{~km}$ of the wake of a $1 \mathrm{~km}$ comet nucleus, computed with a resolution at the nucleus of $R 50(10 \mathrm{~m})$ and in the tail of $100 \mathrm{~m}$. The bottom of the grid lies $153 \mathrm{~km}$ below the 1 bar pressure level. The variables shown are the $\log$ of density, the specific energy (roughly equivalent to temperature), and the concentration of comet material. In each case, white is highest and black is lowest. This model is equivalent to the incompressible R50 model of Figure 6, except for the much larger grid. 


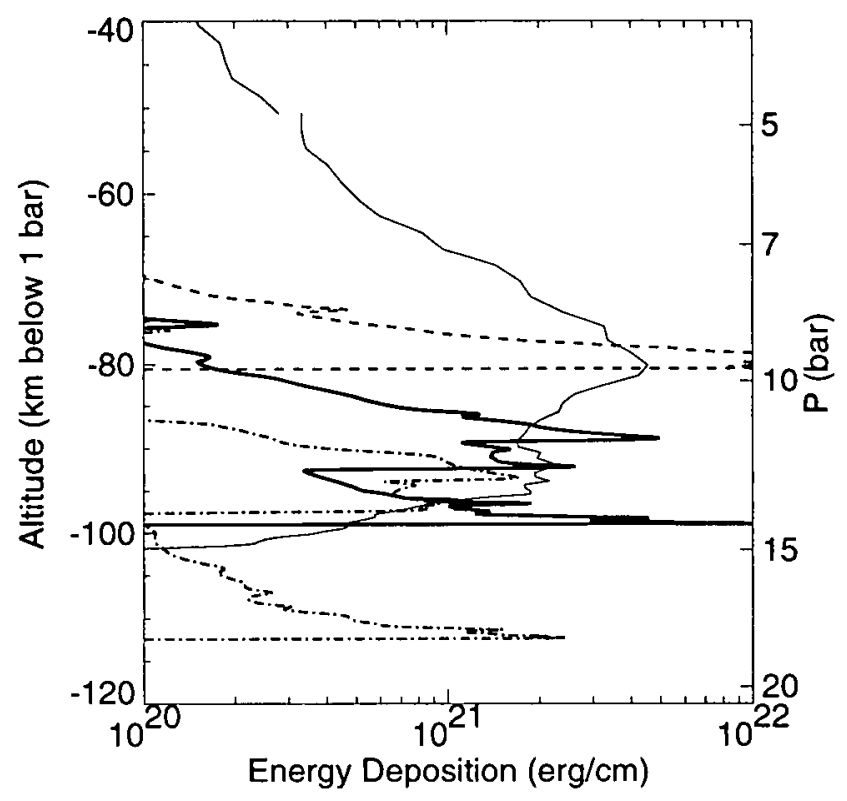

FIguRE 8. Profiles of thermal energy deposited in the atmosphere at times separated by one second (dashed and solid lines), compared to instantaneous energy loss by the comet nucleus (dotted line). The lowest peak in each of the thermal energy curves corresponds to the bow shock, while the higher one is the region of peak energy deposition, which can be seen moving downwards. Note that energy starts flowing off the sides of the grid at the altitude of peak energy deposition (see Fig. 7) after the second curve, so the peak energy is artificially low in the third curve.

tation of this figure is, unfortunately, complicated by the narrow radial extent of the grid, as the developing blast wave runs off the grid sideways, carrying a fair amount of energy with it. However, the downward advection of the energy peak can be seen. It appears that, in this high resolution model, the peak moves down about $30 \mathrm{~km}$ from the altitude predicted by the pancake model. This is within $20 \mathrm{~km}$ of the results obtained by the Sandia group. I believe that the remaining difference comes from the lack of resolution in the wake in the Sandia computation. The low resolution tends to suppress shear instabilities that will act to slow the downward moving wake. Nevertheless, the results are close enough that $I$ conclude that we have reached agreement on the question of the altitude of energy deposition.

Another issue illustrated by this model is that the comet material ends up at the very highest temperatures, so it will not be confined in the deep atmosphere, as suggested by the Sandia group, Takata et al. (1994), and others, but will rise with the plume. At least half the mass of cometary material at the end of the computation has stopped moving downward or already begun to rise.

The filamentary distribution of high-temperature cometary material seen in Figure 7 suggests that its high temperatures would not be captured at lower numerical resolution. This becomes an important issue, as discussed in the next section. Every computational model published, aside from my own, attempts to directly transfer the numerical results of entry models to a larger, lower-resolution grid in order to compute the development of the fireball. The interpolation process involved makes it nearly impossible to maintain the high temperatures shown by the high resolution computation shown in Figure 7, and 


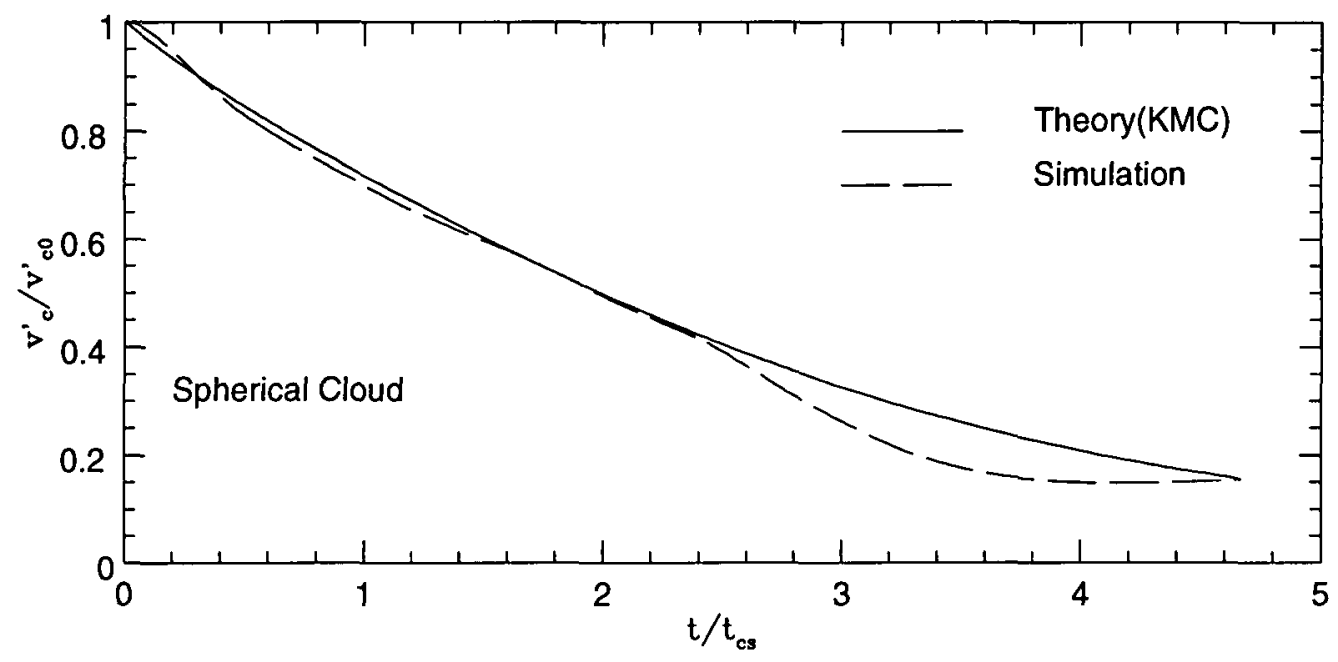

FIGURE 9. Acceleration of a cloud of interstellar gas with density contrast $\chi=10$ hit by a shock wave with Mach number $M=10$. A three-dimensional numerical simulation (dashed line) with resolution of R60 performed by Xu \& Stone (1995) is compared to the analytic pancake model (solid line) of Klein et al. (1994).

so most models do not model the rise of the comet ejecta properly. (The next section discusses the different, but equally large, uncertainties in my own fireball models.)

\subsubsection{Three-dimensional models}

Adequately resolved, three-dimensional, models of the comet entry remain needed. The Sandia group showed an R10 computation (Crawford, this volume), and Takata et al. (1994) have done the R4 computation described above. On the basis of their R10 computation, Crawford speculated that three-dimensional models might show deeper penetration due to non-axisymmetric fluting seen on the edges of the object.

However, adequately resolved, three-dimensional models have been computed for the related problem described in $\S 4.2 .1$, of a supernova shock hitting an interstellar cloud. Xu \& Stone (1995) have computed that problem at a resolution of R60, and find that the acceleration of the cloud has converged well at that resolution, as would be expected from the two-dimensional models. The fluting instability does indeed occur in the material torn off the sides of the cloud, as suggested by Crawford (this volume). The acceleration of the cloud can be compared to the analytic model of Klein et al. (1994), as shown in Figure 9. The behavior of the corresponding two-dimensional model at twice the linear resolution (R120) is shown in Figure 12a of Klein et al. (1994). Comparison of that figure to Figure 9 shows that the three-dimensional model agrees within $3 \%$ with the two-dimensional model; both models diverge slightly from the analytic model, in the same direction. This comparison suggests that three-dimensional effects can also be safely neglected in computing the energy deposition profile of an entering comet nucleus.

\section{Fireball development}

This section treats the fireball resulting from the energy deposition discussed in such detail in the previous section. I discuss the initial conditions, the brightness of the resulting fireball, and the difficulty of determining the energy of the explosion from the height of the observed plumes. 


\subsection{Initial conditions}

The advantages of starting off a computation of the fireball by directly using the final result from an entry computation are obvious. Most modelers have adopted this approach. The difficulties are more subtle. I have taken a different approach that has a complementary set of advantages and problems, namely using the analytic pancake model to generate the initial conditions for the fireball.

Directly using an entry computation for the initial conditions of the fireball requires interpolation from the fine grid used in the entry computation to the coarser grid needed to compute the much larger scale explosion of the fireball. This inevitably requires averaging over much of the detail of the entry computation. Figure 7 shows that important variables such as temperature vary strongly on length scales of a few hundred meters. In my highest resolution, two-dimensional, fireball models, the smallest zones I have used are $0.5 \mathrm{~km}$ across; usually $I$ use at least $1 \mathrm{~km}$ zones. Three-dimensional computations are even more demanding-Crawford et al. (1994) used $5 \mathrm{~km}$ zones for their model of a $3 \mathrm{~km}$ impactor, and only somewhat better (though unspecified) resolution for their model of a $1 \mathrm{~km}$ impactor. The result of this interpolation is to unphysically reduce the temperature of the cometary material and the most strongly shocked jovian atmosphere.

The cometary material in particular should also be hot because its composition gives it a high mean weight per particle $\mu$ compared to the jovian atmosphere; the temperature of gas shocked to a particular velocity is directly proportional to $\mu$. Reduced temperatures in the plumes result in longer rise times, reduced transport of cometary material, and smaller plumes for the same energy deposition.

Instead, I treated the analytic energy deposition profile as a moving line charge (Zahnle \& Mac Low 1995), which has a similarity solution. (My models of the fireball mostly neglect the correction to the analytic model due to the downward advection of the wake. Preliminary tests show that it does not qualitatively change my conclusions, but it should be included for quantitatively correct results.) I assume that the cometary material is mixed with its own weight of jovian atmosphere when computing densities, compositions, and temperatures.

The radius of the wake left by the moving line charge is a parabola in altitude $z$,

$$
R(z) \approx\left(r_{c}^{2}+\left(\frac{4}{\pi} \frac{(\gamma+1)^{2}(\gamma-1)}{3 \gamma+1}\right)^{1 / 2} \frac{1}{\rho(z)} \frac{d E}{d z} \frac{z-z_{0}}{v_{c}}\right)^{1 / 2}
$$

where $z_{0}$ is the altitude of maximum energy deposition; $r_{c}$ is the radius of the impactor and $v_{c}$ is its velocity. The energy deposition rate $d E / d z$ is computed directly from the pancake model, as described below equation (4.13). The wake is assumed to be well-mixed, so that energy and mass are uniformly distributed across it. In most of our models, I neglected the vertical velocity, since an ideal moving line charge has no momentum. (As discussed in $\S 4.2 .2$, this is somewhat inaccurate, but preliminary work shows only quantitative changes in our conclusions due to it.) The energy density in the wake is then

$$
e(z)=\rho_{a t m}(z) \frac{\eta_{e}}{R^{2}(z)} \frac{d E}{d z}
$$

The wake set up is only few zones wide, so clipping due to the finite size of the zones becomes quite important. I apply fudge factors $\eta_{e}$ and $\eta_{d}$ to bring the total energy and density deposited up to the correct values. The assumption of a moving line charge does not describe the deposition of mass in the wake by the comet. Instead, I assume that all 
the energy deposition comes from cometary material being deposited at rest, so that

$$
\frac{d m}{d z}=2 v^{2} \frac{d E}{d z} \text {. }
$$

This is an approximation, since

$$
\frac{d E}{d z}=\frac{1}{2} v^{2} \frac{d m}{d z}+m v \frac{d v}{d z} .
$$

The first term dominates at high altitude, while the second dominates during the final flare and explosion. Better wake models should take this into account. The mass density in the wake is then

$$
\rho^{\prime}(z)=\rho(z)+\frac{\eta_{d}}{R^{2}(z)} \frac{d m}{d z}
$$

Clearly, this description of the entry wake is even more simplified than that given by direct interpolation of entry models onto a larger grid. However, since it is analytic, it can be easily changed. Even though it gets the details wrong, it can capture the essential characteristics of the entry wake, such as the high temperature of the cometary material. Many different models can be run to understand which features are robust and which highly dependent on the details of the initial conditions, and indeed we have run many models in the course of our research. (See Zahnle \& Mac Low 1994 for an example; since then we have more than tripled the number of models run.)

\subsection{Fireball luminosity}

It is difficult to compute the luminosity of the visible fireball-the optically thick gas emitting in visible light. The area of the emitting surface remains quite small during this period, so radiation carries away only a few percent of the total energy, and conservation laws cannot be called upon to help constrain the answer. Instead, a direct computation of the opacity of the hot gas (most likely due to $\mathrm{H}^{-}$) must be done, which involves a careful model of the microphysics. Computing the luminosity of the infrared fireball has similar problems, compounded by our even poorer understanding of the formation of infrared opacity sources (most likely dust).

The connection to the observations appears qualitatively clear, even if it remains quantitatively opaque. The Galileo PPR observations begin when the entering object explosively expands at the altitude of peak energy deposition, as discussed in $\S 3$, with a contribution from the hot, expanding wake above. As viewed from Earth, this occurs while the fireball is still behind the limb of the planet. The fireball is then observed by Galileo to rise, expand and adiabatically cool. By the time the observed fireballs rise over the limb of the planet into sight from the Earth, they have cooled enough that they emit strongly in the near-IR, but not in the visible, producing the peak designated P2 in the IR light curves (Nicholson, this volume).

Ahrens et al. (1994) attempted to compute the visible opacity of the fireball by using an arbitrary but plausible grey opacity for their models of the fireball. They showed that, for a $1 \mathrm{~km}$ object with energy of $10^{28} \mathrm{ergs}$, the visible light fireball should have been easily observable from the Earth as it rose from behind the limb. This conclusion appears to be fairly robust, since Zahnle \& Mac Low (1995) arrived at a similar result by assuming that cometary material with solar abundances of all elements except $\mathrm{H}$ and He was initially mixed equally by mass with clean jovian atmosphere. They followed the advection of the cometary material using a tracer field, and computed the temperature and $\mathrm{H}^{-}$opacity by solving a Saha equation including the easily ionized metals that can provide the free electrons necessary to produce the $\mathrm{H}^{-}$ions. Zahnle \& Mac Low (1995) present visible light curves of the fireball, as viewed from the side, including the effects 


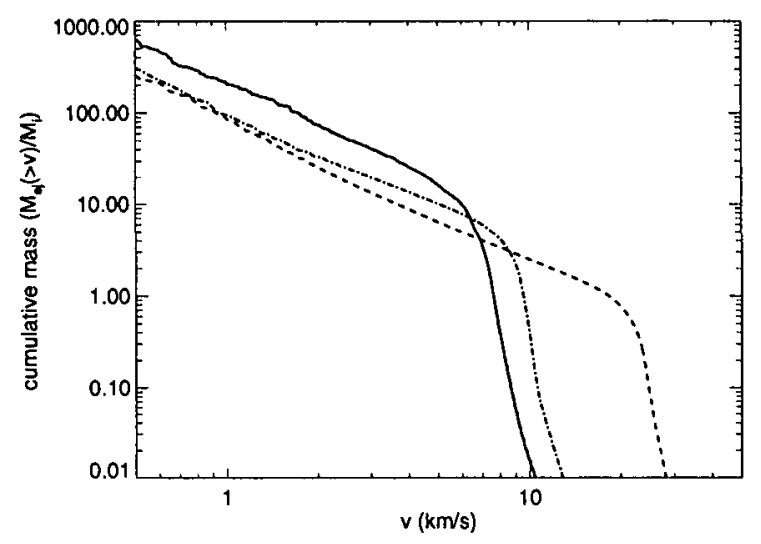

Figure 10. Cumulative mass above each velocity as a function of velocity for three different models with energy of $2 \times 10^{27}$ ergs. One model uses the standard initial conditions described in $\$ 5.1$ (dash-dotted line), one model reduces the total density in the wake by a factor of four, increasing the temperature (dashed line), and one model gives the initial wake a downward velocity of $20 \mathrm{~km} \mathrm{~s}^{-1}$ (solid line).

of the horizon. The lack of a visible fireball observed from the ground therefore suggests that the impacting objects had energies significantly less than $10^{28}$ ergs.

I note that $H S T$ did observe an extremely faint visible fireball almost lost in the glare from the limb (Hammel, this volume). The Galileo $P P R$ result that the visible fireball was only about $10 \%$ of the luminosity of Jupiter (Chapman, this volume) also supports the conclusion that the fireballs were far fainter than expected for $1 \mathrm{~km}$ objects with density of ice.

\subsection{Plume heights}

A number of groups have attempted to calibrate the size of the impactors by comparing the observed heights of the plumes to numerical models, including Takata, Ahrens, \& Harris (1995), Crawford et al. (1995), and Shoemaker et al. (1995). These efforts have two problems, one minor, and one major. The minor problem is that the plumes are observed in sunlight reflecting off of dust, but no model exists for dust formation that can give a prediction for what density of gas will form enough dust to be observed. The major problem is that the velocity of upward expansion of the plume appears quite sensitive to the details of the initial conditions.

These problems can be demonstrated with fireball models using the initial conditions described above. The plume review also uses these models to calibrate the simple analytic models described there. These models use a ratioed grid in cylindrical coordinates, with the highest resolution region ( $1 \mathrm{~km}$ zones) extending from $-150 \mathrm{~km}$ to $100 \mathrm{~km}$ above the one bar level, and for $75 \mathrm{~km}$ radially. The size of the zones then increases by $3 \%$ per zone until the zone size reaches $10 \mathrm{~km}$, and then remains constant to the edges of the grid at an altitude of $3001 \mathrm{~km}$ and a radius of $5076 \mathrm{~km}$, for a total grid size of just under 0.4 megazones. The adiabatic constant $\gamma=1.4$. The model with energy of $2 \times 10^{27}$ ergs that I will pay most attention to has an impactor with density $\rho_{c}=1 \mathrm{~g} \mathrm{~cm}^{-3}$, diameter $600 \mathrm{~m}$, and altitude of maximum energy deposition $25 \mathrm{~km}$ below 1 bar. Clipping requires that I set $\eta_{e}=2.5$ and $\eta_{d}=1.7$ to reach the correct input energy and mass. This particular model is also examined in detail in the plume review.

Let us examine the plume on its way up. Note that there is no sharp line between the end of the fireball phase and the beginning of the plume phase, though practically 
speaking, it occurs when the rising gas no longer emits detectable thermal emission, and only reflects sunlight. (At least until it falls back and gets reheated, as the plume review discusses.) A useful way of displaying the results of these models is by measuring the cumulative mass above each velocity, as shown in Figure 10. This figure shows the results from three different runs with energies of $2 \times 10^{27} \mathrm{ergs}$, but with slight variations on the initial conditions. One run uses exactly the initial conditions described in $\S 5.1$ and above. The next run uses those initial conditions, but with the density in the wake, $\rho^{\prime}(z)$ in equation (5.19), reduced by a factor of four, increasing the temperature in the wake without changing the total energy. The third run uses the initial conditions of $\$ 5.1$, but gives the wake a uniform downward motion of $20 \mathrm{~km} \mathrm{~s}^{-1}$, somewhat faster than the energy actually appears to be advected in Figure 8 . This last run represents the maximum effect that the downward motion of the wake should have, both because it is moving downward so fast, and because the initial wake is quite underresolved, suppressing shear instabilities that would otherwise slow the wake.

Figure 10 shows that the main difference between the three different sets of initial conditions is the position of the knee where each of the cumulative velocity curves bends over. The shapes of the three curves remain quite constant, suggesting that this is a robust result. Zahnle \& Mac Low (1995) discuss in detail why a power-law distribution of ejecta such as this is expected from a blowout. It is tempting to treat the knee as the natural edge of the plume, since relatively little gas rises faster than this velocity. The presence of the knee in all three models suggests that the problem of not knowing where the plume ends may indeed not be a major one, though dust formation models will be required to answer the question definitively.

The position of the knee, on the other hand, clearly depends sensitively on the details of the initial conditions. In order to reach the heights observed of about $3000 \mathrm{~km}$ above the 1 bar level (Hammel, this volume), a vertically rising plume must travel at $12 \mathrm{~km} \mathrm{~s}^{-1}$, while a plume rising at $45^{\circ}$ must travel at $17 \mathrm{~km} \mathrm{~s}^{-1}$. The Sandia group has shown that, in fact, the plume does tend to follow the entry path initially. However, Jessup, Clarke \& Hammel showed in a poster at this conference that the plumes did not travel sideways at $12 \mathrm{~km} \mathrm{~s}^{-1}$, so the plumes may begin to rise more vertically once they have left the atmosphere.

Clearly, gas in these models can reach the correct velocities, but calibration appears very difficult. I believe that it is quite likely that, in the short term, modelers may be forced to calibrate their models against the plume height observations, rather than being able to predict them, because of the difficulty of correctly modeling the details of things like the equation of state of jovian air mixed with varying amounts of cometary material. Therefore, I believe that no solid information about the size of the impactors can currently be derived from models of the plume height.

\section{Plume bounces}

I am now going to skip lightly over the period of plume fallback that generates most of the observed IR, the main event, in Nicholson's terminology (this volume), and the chemistry of the plume and spots, all of which is discussed in the plume review. However, the last act of the plume dynamics deserves attention. The ballistic plume rises to its peak height, where all its kinetic energy has been converted into gravitational potential energy, and then falls, regaining kinetic energy until it hits the atmosphere at the same velocity it was ejected, converting its energy back to thermal energy in a shock wave. The strong IR radiation of the main event carries away much of this energy, but not all of it. The remaining energy gets converted back into kinetic energy by the expansion 
of the shocked, high-pressure, layer of plume material back up into space when infalling plume material no longer confines it. This material eventually comes crashing back down, releasing further IR radiation.

I noted these bounces in simulations well before the event. For example, Figure 11 shows a plot of material moving back up shown in my presentation at the Maryland Workshop in 1994 January. I discounted the appearance of bounces in the models, expecting the radiative cooling to be strong enough to suppress them, until I saw the first light curves from Nicholson (this volume) and Graham et al. (1995) that clearly show two bounces ten and twenty minutes after the main event. The alert reader may note what neither I nor anyone else realized at the time, namely that if the radiative cooling were strong enough to suppress the bounces, it would produce strong enough emission to be easily observable, as, indeed, it was. This, in my opinion, was the fundamental error that prevented a clear prediction of the main event from being made well before the impacts.

A one-dimensional model of the bounces, including radiative cooling, was presented by Deming et al. at this meeting. In this model, the infalling plume was modeled as a simple slab of gas falling from $3000 \mathrm{~km}$ onto the jovian atmosphere, and the radiative emission was modeled with a grey opacity of $0.4 \mathrm{~cm}^{-2} \mathrm{~g}^{-1}$. Figure 12 shows the emission from the series of bounces. The timing and relative brightness appear to match the observations convincingly.

\section{Conclusions}

In this article, I have presented a critical review of models of the tidal breakup, entry, and fireball, as these provide the basis for any deduction from the observations of the size and energy of the objects. I believe that the areas that are well understood are the initial entry and energy deposition, the behavior of the plume once it has been launched, and, although controversy continues, the tidal breakup models. The area that currently needs further work is the translation of the models for energy deposition into satisfactory initial conditions for models of the initial development of the fireball from the entry wake.

The evidence for the size and energy of the objects can be broken into three categoriesevidence for shallow penetration of the jovian atmosphere, evidence for small impactor energies, and direct evidence for small impactor sizes.

Observations of sulfur and water appear to show that the explosions occurred above the water clouds. Zahnle et al. (1995) show that the observations of large amounts of $\mathrm{S}_{2}$ and $\mathrm{CS}_{2}$ and small amounts of $\mathrm{SO}_{2}$ can be best explained by shock chemistry in dry jovian air, perhaps mixed with small amounts of cometary oxygen. Direct observations of $\mathrm{H}_{2} \mathrm{O}$ and $\mathrm{CH}_{4}$ in the $\mathrm{G}$ and $\mathrm{K}$ impact sites by Bjoraker et al. (1995) gave a ratio of around unity, which they interpreted as an observation of cometary water in quantities of order $10^{12} \mathrm{~g}$, equivalent to spheres of ice with diameters of order $100 \mathrm{~m}$, clearly a lower limit to the impactor sizes. (Note that Lellouch, in this volume, interprets CO observations to derive objects as large as $1 \mathrm{~km}$ at densities of $0.5 \mathrm{~g} \mathrm{~cm}^{-3}$, at the upper limit of our range.) Radio observations of thermal emission at 3 and $6 \mathrm{~cm}$ show no perturbations at the 5 bar level where the water clouds are expected to lie (Grossman et al. in a poster at this meeting), also suggesting energy deposition higher in the atmosphere, though beam dilution could hide small perturbations. Models of the entry show that only small impactors will deposit their energies at such high altitudes. 


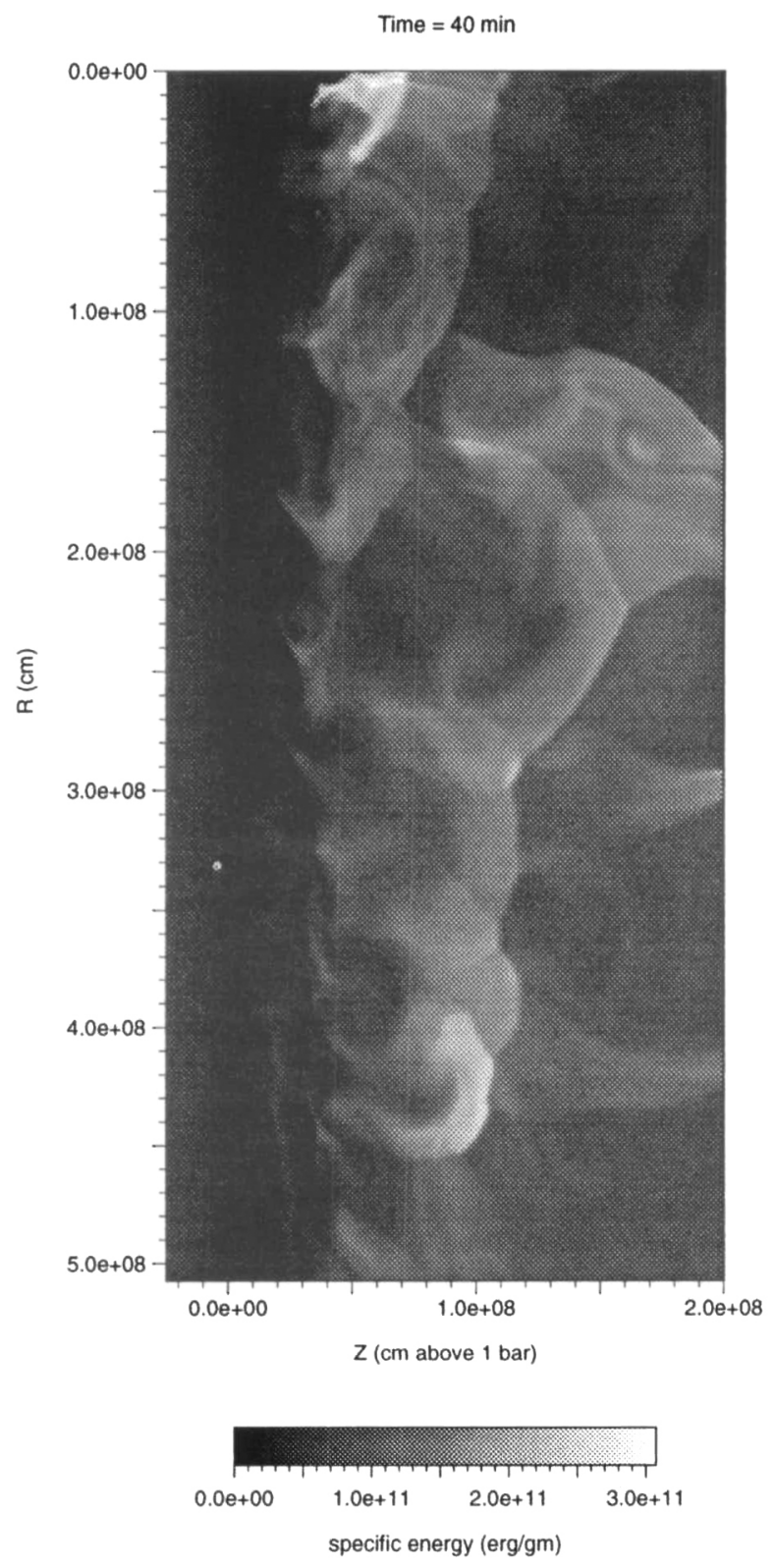

FIGURE 11. Specific energy (roughly equivalent to temperature) of gas at a time of 40 minutes after impact for the plume from a $1 \mathrm{~km}$ object in the absence of radiative cooling. Note that the center of the planet is to the left, and that the temperature minimum in the tropopause may just be visible at the left of the plot as a darker line. Regions of high specific energy trace hot shock waves as material bounces up. 


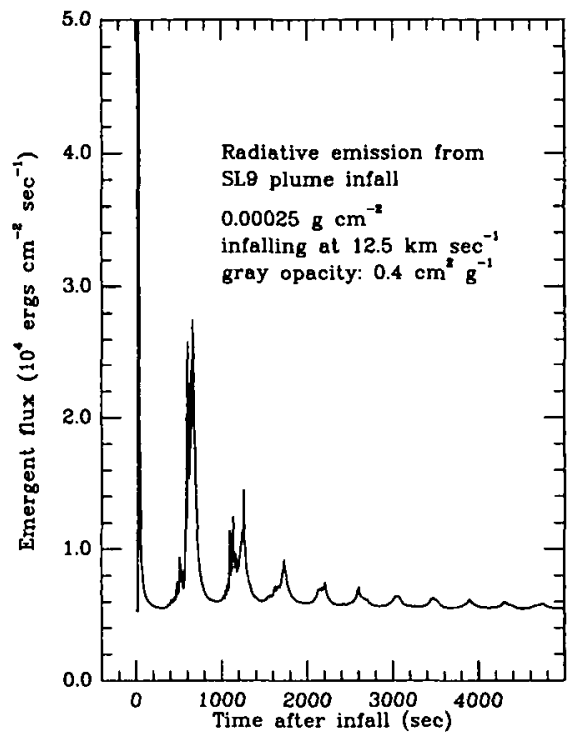

Figure 12. Model by Deming et al. of the emission from the bouncing plume, using a one-dimensional model with an infalling slab of gas and the parameters noted. Note that the emission from the first bounce goes far off scale on this linear plot. The second and third bounces were observed.

The luminosity of the visible fireballs and the infrared main events suggest low impactor energies, as does the lack of observed seismic waves. Kilometer-sized impactors with density close to $1 \mathrm{~g} \mathrm{~cm}^{-3}$ would have produced visible fireballs with luminosity comparable to Jupiter's as observed from Galileo, and easily visible from the Earth as they rose above the horizon ( $₫ 5.2$; Zahnle \& Mac Low 1995). The luminosities and lifetimes of the infrared main events are best fit by semi-analytic plume models with low energies as well; for example, the $\mathrm{R}$ impact is best fit by an impact with energy $6 \times 10^{26} \mathrm{erg}$ (the plume review; Zahnle \& Mac Low 1995).

Attempts were made to detect seismic waves generated by the impacts by Marley et al. (1994) and Logonné et al. (1994). Neither group was successful, giving upper limits on the energy of the impactor of under $10^{28} \mathrm{erg}$. These limits depend on the partitioning of energy into the seismic modes, which is not known well; however neither group assumed more than $30 \%$ efficiency. Higher efficiencies might be expected if most of the impact energy were buried in the atmosphere, which would lower the energy limits even further. These limits will probably also be reduced as the search images are processed more carefully.

Self-gravitating cluster models of the tidal breakup by Asphaug \& Benz (1994) and Solem (1994) can explain the observed length and position angle of the comet train, and the number of fragments in it. These models conclude that the parent body had a diameter of $1.5 \mathrm{~km}$ and a density of $0.6 \mathrm{~g} \mathrm{~cm}^{-3}$, suggesting that the fragments have radii in the half-kilometer range. Another piece of circumstantial evidence for a small parent body is the estimate of the total volume of dust in the stratosphere by West et al. (1995), who derived a mass equivalent to a sphere of diameter $1 \mathrm{~km}$ the day after the last impact. 
From these various pieces of evidence, it appears that the most coherent picture emerges if the largest nuclei of Comet Shoemaker-Levy 9 had diameters under $1 \mathrm{~km}$, densities of order $0.5 \mathrm{~g} \mathrm{~cm}^{-3}$, and kinetic energies of order $10^{27} \mathrm{ergs}$.

All the original work presented here was done in collaboration with Kevin Zahnle. The section on tidal breakup models derives from a draft manuscript provided by Erik Asphang (Asphang and Beng 1995). Computations were performed at the Pittsburgh Supercomputing Center, and at the National Center for Supercomputing Applications, using software provided by the Laboratory for Computational Astrophysics. This work was supported by the NSF Aeronomy Program under grant number AST93-22509, and by the NASA programs in Exobiology and Astrophysical Theory. Too many people to list have been extremely generous in sharing results and preprints prior to publication, an openness that has been one of the joys of working on this problem.

\section{REFERENCES}

Ahrens, T. J., Takata, T., \& O'Keefe, J. D. 1994 Radiative signatures from impact of comet Shoemaker-Levy 9 on Jupiter. Geophys. Res. Lett., 21, 1551-1553.

Asphaug, E. \& Benz, W. 1994 Density of comet Shoemaker-Levy 9 deduced by modelling breakup of the parent 'rubble pile'. Nature, 370, 120-124.

AsPhaug, E. \& BENZ, W. 1995 The tidal disruption of strengthless bodies: Lessons from comet Shoemaker-Levy 9. Icarus, submitted.

Biberman, L. M., Bronin, S. Ya., \& Brykin, M. V. 1980 Moving of a blunt body through a dense atmosphere under conditions of severe aerodynamic heating and ablation. Acta Astronaut., 7, 53-65.

Bjoraker, G. L., Stolovy, S. R., Herter, T. L., Gull, G. E., \& Pirger, B. E. 1995 Detection of water after the collision of fragments $G$ and $K$ of comet Shomeaker-Levy 9 with Jupiter. Icarus, submitted.

Boslough, M. B., Crawford, D. A., Robinson, A. C., \& Trucano, T. G. 1994 Mass and penetration depth of Shoemaker-Levy 9 fragments from time-resolved photometry. Geophys. Res. Lett., 21, 1555-1558.

Boslough, M. B., Crawford, D. A., Trucano, T. G., \& Robinson, A. C. 1995 Numerical modeling of Shoemaker-Levy 9 impacts as a framework for interpreting observations. Geophys. Res. Lett., 22, 1821-1824.

Bronshten, V. A. 1983 Physics of Meteoric Phenomena Reidel.

ChandrasekHar, S. 1961 Hydrodynamic and hydromagnetic stability. Dover.

Chevalier, R. A. \& Sarazin, C. L. 1994 Explosions of infalling comets in Jupiter's atmosphere. Astrophys. J., 429, 863-875.

Chyba, C. F., Thomas, P. J., \& Zahnle, K. J. 1993 The 1908 Tunguska explosion: Atmospheric disruption of a stony asteroid. Nature, 361, 40-44.

Crawford, D. A. Boslough, M. B., Trucano, T. G., and Robinson, A. H. 1994 The impact of comet Shoemaker-Levy 9 on Jupiter. Shock Waves, 4, 47-50.

Crawford, D. A. Boslough, M. B., Trucano, T. G., and Robinson, A. H. 1995 The impact of periodic comet Shoemaker-Levy 9 on Jupiter. Int. J. of Impact Engin., in press.

Dobrovolskis, A. R. 1990 Tidal disruption of solid bodies. Icarus, 88, 24-38.

Field, G. B. \& Ferrara, A. 1995 The behavior of fragments of comet Shoemaker-Levy 9 in the atmosphere of Jupiter. Astrophys. J., 438, 957-967.

Graham, J. R., de Pater, I., Jernigan, J. G., Liu, M. C., \& Brown, M. E. 1995 The fragment R collision: W. M. Keck telescope observations of SL9. Science, 267, 1320-1323.

HahN, J. M. \& Rettig, T. W. 1995 Jeans instability in comet Shoemaker-Levy 9. J. Geophys. Res.-Planets, submitted. 
Klein, R. 1., McKee, C. F., \& Colella, P. 1994 On the hydrodynamic interaction of shock waves with interstellar clouds. I. Nonradiative shocks in small clouds. Astrophys. J., 420, 213-236.

Logonné, P., Billebaud, F., Vauglin, I., Merlin, P., Sybille, F., Mosser, B., Lagage, P. O., Gautier, D., \& Drossart, P. 1994 Seismic waves generated by the SL-9 impact. Bull. Amer. Astron. Soc., 26, 1580.

MaC Low, M.-M. \& ZaHNLE, K. 1994 Explosion of comet Shoemaker-Levy 9 on entry into the jovian atmosphere. Astrophys. J. 434, L33-L36.

Marley, M. S., Dayal, A., Deutsch, L. K., Fazio, G. G., Hoffmann, W. F., Hora, J. L., Hunten, D. M., Sprague, A. L., Sykes, M. V., Walter, C., \& Wells, K. W. 1994 A search for seismic waves launched by the impact of comet Shoemaker-Levy 9. Bull. Amer. Astron. Soc., 26, 1580.

Moran, B. \& Tipton, R. 1993 Simulation of a comet impact on Jupiter. Eos, 74, 43, 389.

READS, K. I. 1984 Experimental investigation of turbulent mixing by Rayleigh-Taylor instability. Physica, 12D, 45-58.

Richardson, D., Asphaug, E., \& BenNer, L. 1995 Precise orbital integration of the selfgravitational clustering of SL9 debris. Bull. Amer. Astron. Soc., in press.

Schenk, P., Asphaug, E., McKinnon, W., Melosh, H. J., \& Weissman, P. 1995 Cometary nuclei and tidal disruption: The geologic record of crater chains on Callisto and Ganymede. Icarus, submitted.

Scotti, J. V., \& Melosh, H. J. 1993 Tidal breakup and dispersion of P/Shoemaker-Levy 9: Estimate of progenitor size. Nature, 365, 7333.

Sekanina, Z. 1993 Disintegration phenomena expected during collision of comet ShoemakerLevy 9 with Jupiter. Science, 262, 382-387.

Sekanina, Z. 1996 Preprint Astronomy \& Astrophysics, in press.

Seranina, Z., Chodas, P. W., \& Yeomans, D. K. 1994 Tidal disruption and the appearance of periodic comet Shoemaker-Levy 9. Astron. Astrophys., 289, 607-636.

Shoemaker, E. M., Hassig, P. J., \& Roddy, D. J. 1995 Numerical simulations of the Shoemaker-Levy 9 impact plumes and clouds: A progress report. Geophys. Res. Lett., 22, 1825-1828.

Solem, J. 1994 Density and size of comet Shoemaker-Levy 9 deduced from a tidal breakup model. Nature, 370, 349-351.

Sridhar, S., \& Tremaine, S. 1992 Tidal disruption of viscous bodies. Icarus, 95, 86-99.

Svetsov, V. V., Nemtchinov, I. V., \& Teterev, A. V. 1995 Disintegration of large meteoroids in Earth's atmosphere: Theoretical models. Icarus, 116, 131-153.

Stone, J. M. \& Norman, M. L. 1992 Zeus-2D: A radiation magnetohydrodynamics code for astrophysical flows in two space dimensions. I. The hydrodynamic algorithms and tests. Astrophys. J. Supp., 80, 753-790.

Takata, T., O'Keefe, J. D., Ahrens, T. J., \& Orton, G. S. 1994 Comet Shoemaker-Levy 9: Impact on Jupiter and plume evolution. Icarus 109, 3-19.

Takata, T., Ahrens, T. J., Harris, A. W. 1995 Comet Shoemaker-Levy 9: Fragment and Progenitor Energy. Geophys. Res. Lett., 22, 2429-2432.

VAN LeER, B. 1977 Towards the ultimate conservative difference scheme. IV. A new approach to numerical convection. J. Comp. Phys., 23, 276-299.

VickerY, A. M. 1993 Numerical simulation of a comet impact on Jupiter. Eos, 74, 43, 391.

Weaver, H. A., Feldman, P. D., A'Hearn, M. F., \& Arpigny, C. 1994 Hubble Space Telescope observations of comet P/Shoemaker-Levy 9 (1993e). Science, 263, 787-791.

Weidenschilling, S. J. 1994 Origin of cometary nuclei as 'rubble piles'. Nature, 368, 721-723.

West, R. A., Karkoschka, E., Friedson, A. J., Seymour, M., Baines, K. H., \& Hammel, H. B. 1995 Impact debris particles in Jupiter's stratosphere. Science, 267, 1296-1301.

Wingate, C. A., Hoffman, N. M., \& Stellingwerf, R. F. 1994 SPH calculations of comet Shoemaker-Levy 9/Jupiter impact. Bull. Amer. Astron. Soc., 26, 879-880. 
XU, J. \& Stone, J. M. 1995 The hydrodynamics of shock-cloud interactions in three dimensions. Astrophys. J., in press.

Yabe, T., Xiao, F., Zhang, D., Sasaki, S., Abe, Y., Kobayashi, N., \& Terasawa, T. 1994 Effect of EOS on break-up of Shoemaker-Levy 9 entering jovian atmosphere. J. Geomag. Geoelectr., 46, 657-662.

YouNGS, D. L. 1984 Numerical simulation of turbulent mixing by Rayleigh-Taylor instability. Physica, 12D, 32-44.

ZAHNLE, K. J. 1992 Airburst origin of dark shadows on Venus. J. Geophys. Res., 97, 1024310255 .

ZahNLE, K. \& MAC Low, M.-M. 1994 The collision of Jupiter and comet Shoemaker-Levy 9. Icarus, 108, 1-17.

ZAHNLE, K. \& MAC Low, M.-M. 1995 A simple model for the light curve generated by a Shoemaker-Levy 9 impact. J. Geophys. Res.-Planets, in press.

Zahnle, K., MaC Low, M.-M., Lodders, K., \& Fegley, B. 1995 Sulfur chemistry in the wake of comet Shoemaker-Levy 9. Geophys. Res. Lett., 22, 1593-1596. 